55
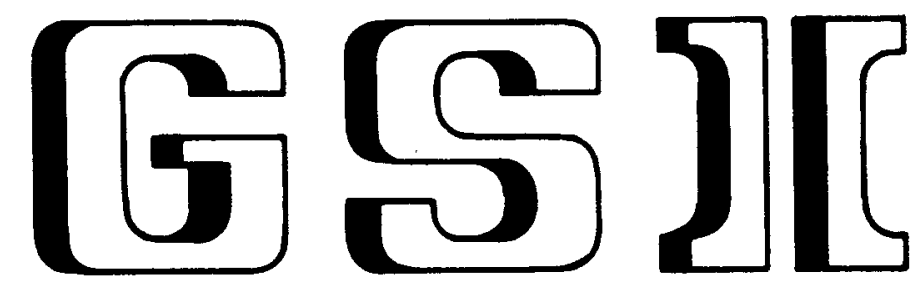

GSI-Preprint-95-58 OCTOBER 1995

APPLICATION OF LOW TEMPERATURE CALORIMETERS FOR PRECISE LAMB SHIFT MEASUREMENTS ON HYDROGEN-LIKE VERY HEAVY IONS

P. EGELHOF, H.F. BEYER, D. MCCAMMON, F.v. FEILITZSCH, A.v. KIENLIN, H.-J. KLUGE, D. LIESEN, J. MEIER, H.S. MOSELEY, T. STÖHLKER

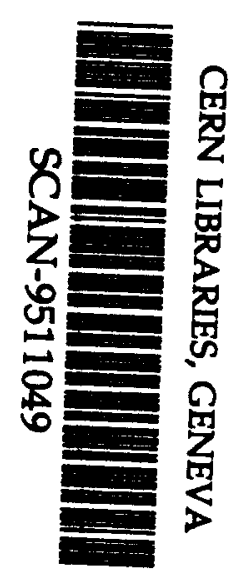

(Contribution to the 'Sixth int. Workshop on Low Temperature Detectors', Beatenberg/ Interlaken, Switzerland, Aug.28-Sept.1, 1995)

Gesellschaft für Schwerionenforschung $\mathrm{mbH}$ Postfach 110552 - D-64220 Darmstadt · Germany 


\title{
Application of Low Temperature Calorimeters for Precise Lamb Shift Measurements on Hydrogen-like Very Heavy Ions
}

P. Egelhof ${ }^{1}$, H.F. Beyer ${ }^{1}$, D. McCammon ${ }^{2}$, F.v. Feilitzsch ${ }^{3}$, A.v. Kienlin ${ }^{1}$, H.-J. Kluge ${ }^{1}$, D. Liesen ${ }^{1}$, J. Meier ${ }^{1}$, H.S. Moseley ${ }^{4}$ and T. Stöhlker ${ }^{1}$

1. Gesellschaft für Schwerionenforschung (GSI), Postfach 110552, D-64220 Darmstadt, Germany

2. Department of Physics, University of Wisconsin, Madison, WI 53706, USA

3. Technische Universität München, D- 85747 Garching, Germany

4. NASA/ Goddard Space Flight Center, Greenbelt, MD 20771, USA

\begin{abstract}
The precise determination of the $2 \mathrm{~s}_{1 / 2}, 2 \mathrm{p}_{1 / 2}, 2 \mathrm{p}_{32} \rightarrow 1 \mathrm{~s}_{1 / 2} \mathrm{X}$-ray transitions in hydrogen-like very heavy ions such as ${ }^{208} \mathrm{~Pb}^{81+}$ or ${ }^{238} \mathrm{U}^{91+}$ provides a sensitive test of $\mathrm{QED}$, especially of higher order contributions to the self-energy which are not accessible by alternative methods. Such measurements became possible recently by X-ray spectroscopy using highly charged ions stored and cooled in heavy ion storage rings. A better energy resolution of the X-ray detector is most essential in order to improve the experimental accuracy, which is presently about one order of magnitude worse than the theoretical uncertainty. An energy resolution of $\Delta \mathrm{E}=30 \mathrm{eV}-50 \mathrm{eV}$ for $\mathrm{E}_{\gamma}=50 \mathrm{keV}-100 \mathrm{keV}$, and a photopeak efficiency above $30 \%$ may be provided by a low-temperature calorimeter. It will be constructed on the basis of already existing arrays of silicon microcalorimeters, but with larger volume and high $\mathrm{Z}$ absorbers. Such a detector will allow a more precise determination of the 1s- Lamb shift and for the first time the direct investigation of the $2 s$ - Lamb shift in hydrogen-like heavy ions. In the present contribution the experimental scenario for QED experiments at storage rings, the present status of experimental and theoretical investigations and the design of an appropriate low temperature detector are discussed.
\end{abstract}




\section{Motivation}

About fifty years ago it became evident that for small distances between electric charges the classical Coulomb interaction potential is not completely correct. The precise experimental test of the theoretical predictions by quantum electrodynamics (QED) then developed is still - at least for high $\mathrm{Z}$ systems - one of the outstanding and most challenging problems of atomic physics. The hydrogen atom or hydrogen-like ions are testing grounds of QED since these are the simplest atomic systems. Here the QED corrections give rise to the so called Lamb shift which is a small deviation of the binding energies from those predicted by the correct relativistic Dirac-Coulomb energy (see fig.1). It is largest for

s- states, where the binding energies will be lowered by an amount of: $L_{n s}=\frac{\alpha}{\pi} \frac{(Z \alpha)^{4}}{n^{3}} F(Z \alpha) m_{0} c^{2}$; here $\alpha$ is the fine structure constant, $Z$ the atomic number of the ion, and $m_{0} c^{2}$ the electron rest mass. The function $\mathrm{F}(\mathrm{Z} \alpha)$ comprises all the necessary correction terms and can be approximated by a $(Z \alpha)^{\mathrm{n}}$ power expansion. The largest contribution is the self- energy correction (except for myonic atoms where the vacuum polarization is dominant), followed by the vacuum polarization. For the heaviest ions also the nuclear size correction has to be considered.

For the hydrogen atom the ,classical Lamb shift“, namely the $2 s_{12}-2 p_{1 / 2}$ energy level splitting, was determined experimentally to an extraordinary precision of about $10^{-5}$ [1]. Whereas in light sytems the higher order effects are almost negligible, they increase strongly with higher $\mathrm{Z}$ and are the most important QED contributions for the heaviest ions like $\mathrm{U}^{91+}$. On the other hand the series expansion of $\mathrm{F}(\mathrm{Z} \alpha)$ becomes most critical for the heaviest systems, where $\mathrm{Z} \alpha$ approaches values close to unity. Therefore a precise determination of the Lamb shift in hydrogen-like very heavy ions represents one of the most sensitive tests of QED in strong electromagnetic fields.

\section{Experimental Scenario and Present Status of Experiment and Theory}

The level scheme of the $\mathrm{U}^{91+}$ - ion is displayed in fig.1. The binding energy of the 1s-energy level is about $132 \mathrm{keV}$, thus yielding transition energies for the Lyman- $\alpha$-lines of about $100 \mathrm{keV}$. Whereas the $2 s$ - state is metastable in very light systems, the transition rate of the $\mathrm{M} 1$ - transition increases with $\mathrm{Z}^{10}$, and therefore the 2s-state decays prompt in heavy systems. The Lamb shift is predicted to be $\mathrm{L}_{1 \mathrm{~s}}=458 \mathrm{eV}$ for the $1 \mathrm{~s}-$ level and $\mathrm{L}_{2 \mathrm{~s}}=75 \mathrm{eV}$ for the $2 \mathrm{~s}$ - level, respectively. For an overview on the experimental results obtainted up to now for the Lamb shift in hydrogen-like ions the reader is referred to $[2,3]$.

The experimental situation for the investigation of the Lamb shift in the heaviest ions has improved considerably since the new facilities of the GSI Darmstadt [4], namely the Heavy Ion Synchrotron (SIS) and the Experimental Storage Ring (ESR) became available. The SIS accelerator provides the acceleration of all ion species up to uranium to energies up to $1-2 \mathrm{GeV} / \mathrm{u}$. Since an energy 
of $\mathrm{E} \geq 200 \mathrm{MeV} / \mathrm{u}$ is required for production of bare heavy ions, the SIS accelerator is quite appropriate for atomic physics experiments on highly charged ions. The ESR storage ring provides high beam quality by electron cooling, energy tuning and an internal gas target or an electron target for the population of excited $2 \mathrm{~s}$ - and $2 \mathrm{p}$ - states.

In fig. 2 the experimental setup for Lamb shift measurements at the internal ESR gas target and at the electron cooler is displayed. A beam of bare $\mathrm{U}^{92+}$ - ions at $\mathrm{E}=295 \mathrm{MeV} / \mathrm{u}$ is injected into the ESR and cooled in the electron cooler. The capture of an electron from the electron target or the gas jet target and the emitted X-rays are detected by Ge- detectors and position sensitive particle detectors (PSD) for the down-charged hydrogen-like $\mathrm{U}^{91+}$ - ions.

The latest results on the $Z=79$ and $Z=92$ systems are summarized and compared with theoretical predictions in tab. 1 (taken from [5], see also references in [5]). For all cases the experimental results agree within the error bars with the theoretical predictions, but the experimental errors are about one order of magnitude larger than the theoretical ones. Thus the experimental accuracy has to be improved considerably for a more stringent test of QED. The most crucial experimental errors are on the one hand due to uncertainties in beam velocity and in the observation angle. Both influence the determination of the transition energy in the center-of-mass frame from the measured transition energy via the Doppler shift. On the other hand one major contribution is, however, the poor energy resolution of about $\Delta \mathrm{E} \cong 800 \mathrm{eV}$ for $\mathrm{X}$-ray energies of $50 \mathrm{keV} \leq \mathrm{E}_{\gamma} \leq 150 \mathrm{keV}$ obtained with Ge-detectors, which must be improved to reach an absolute accuracy of about $\Delta \mathrm{E}= \pm \mathrm{leV}$. This was the motivation for the design of a low temperature detector for the present application as discussed in the following section.

\section{Design of a Low Temperature Detector for Lamb shift Experiments with Very Heavy Hydrogen-like Ions}

We plan to replace at least one of the Ge- detectors surrounding the internal gas jet target or the detector at the electron cooler (see fig.2) by a low temperature detector array. With such a new setup we intend to:

- improve the energy resolution by more than one order of magnitude for a better determination of the absolute transition energy,

- to resolve the $2 p_{12} \rightarrow 1 s_{12}\left(L y_{\alpha 1}\right)$ and the $2 s_{12} \rightarrow 1 s_{12}$ (M1) transitions, which would allow for the first time the investigation of the $2 s$-Lamb shift in heavy systems. By such a relative measurement it will be possible to reduce considerably various systematic errors which limit the accuracy of an absolute measurement.

In order to meet these experimental conditions the detector should fulfill the following constraints: 
- energy resolution : $\Delta \mathrm{E}=30 \mathrm{eV}-50 \mathrm{eV}$ for $\mathrm{E}_{\gamma}=50 \mathrm{keV}-100 \mathrm{keV}$ (for backward angles the laboratory energy is down to about $\mathrm{E}_{\gamma}=50 \mathrm{keV}$ due to the Doppler shift).

- detection efficiency : effective area $\geq 100 \mathrm{~mm}^{2}$; absorption efficiency $\geq 30 \%$ for $\mathrm{E}_{\gamma}=50 \mathrm{keV}-100 \mathrm{keV}$.

- signal rise time $\quad: \tau_{\mathrm{R}} \leq 100 \mu \mathrm{sec}$ in order to measure in coincidence with the downcharged heavy ions $(\mathrm{R} \approx 10 \mathrm{kHz}-50 \mathrm{kHz})$.

- signal decay time $: \tau_{\mathrm{F}} \leq 20 \mathrm{msec}$ for sufficiently low pile-up (background rate $\approx$ $10 / \mathrm{sec} \mathrm{mm}^{2}$ ).

- position resolution : $\mathrm{d} \leq 0,25-0,5 \mathrm{~mm}$ (in order to reduce Doppler broadening).

We aim to construct a detector array on the basis of silicon microcalorimeters [6] which were designed for lower $\mathrm{X}$-ray energies $\left(\mathrm{E}_{\gamma} \leq 6 \mathrm{keV}\right)$, and which have already shown excellent energy resolution $(\Delta \mathrm{E}=7.3 \mathrm{eV})$ for this energy range. In order to reach sufficient photopeak efficiency for higher energy $\mathrm{X}$-rays, the absorber should be a high $\mathrm{Z}$ material and should have a volume of $\mathrm{V} \geq 1-2 \mathrm{~mm}^{2} \times 40 \mu \mathrm{m}$. Candidates which fulfill also the condition of low heat capacity as well as rapid and complete thermalisation are HgTe, Sn, Bi and Re. Excellent results have been reported [7] for absorber volumes which almost reach already the above conditions for $\mathrm{HgTe}(\Delta \mathrm{E}=11.5 \mathrm{eV}$ for $\mathrm{V}=$ $0.25 \mathrm{~mm}^{2} \times 12 \mu \mathrm{m}$ and $\left.E_{\gamma}=6 \mathrm{keV}\right)$ and for $\mathrm{Sn}\left(\Delta \mathrm{E}=21.4 \mathrm{eV}\right.$ for $\mathrm{V}=1 \mathrm{~mm}^{2} \times 13 \mu \mathrm{m}$ and $\left.E_{\gamma}=6 \mathrm{keV}\right)$. For $X-$ rays energies above $75 \mathrm{keV}$ Re might be an excellent candidate (due to the $\mathrm{K}$ absorption edge in this energy region), but problems with long tails in the thermal signals, probably due to material impurities have been reported [8]. On the other hand promising results have been presented at this workshop [9] with a $0.5 \times 0.5 \times 0.5 \mathrm{~mm}^{3}$ Re-crystal and operating temperatures above $100 \mathrm{mK}$.

Large solid angle arrays with an effective area of up to $36 \times 1 \mathrm{~mm}^{2}$ have already been demonstrated to work well [10], so we do not expect any principal difficulties when extending the effective area by about a factor of three. However, only little information exists on the performance of low temperature $X$-ray detectors for higher energies. Recent results with $S n$ absorbers with $\Delta E=90 \mathrm{eV}$ for $\mathrm{E}_{\gamma}=32 \mathrm{keV}$ with a not perfectly optimized detector [11], and $\Delta \mathrm{E}=52 \mathrm{eV}$ for $\mathrm{E}_{\gamma}=60 \mathrm{keV}$ using a NTD-thermistor [12] are promising. Here some more systematic work, especially for high Z absorbers is required. In order to meet the geometrical conditions at the ESR we plan to house the detector array in a dilution refrigerator with a side excess of about $50 \mathrm{~cm}$ length. The operating temperature will be about $50-80 \mathrm{mK}$.

The expected performance of such a low temperature detector is summarized in tab. 2 and compared to that of a conventional Ge-detector and of a crystal spectrometer. The latter spectrometer may provide excellent energy resolution but suffers from the poor overall detection efficiency. The calorimeter design combines a good energy resolution with a detection efficiency close to that of a Ge- 
detector. Hence a considerable improvement of the experimental conditions can be expected from the use of calorimetric low temperature detectors for precise Lamb shift measurements on hydrogen-like very heavy ions.

\section{References:}

[1] M. Weitz et al., Phys. Rev. Lett 68 (1992) 1120

[2]P.H. Mokler et al., Phys. Scripta T51 (1994) 28

[3] D. Liesen et al., Comments At. Mol. Phys. 32 (1995) 23

[4] K. Blasche et al., Proc. 4th Europ. Part. Accel. Conf., London, 1994; V. Suller, Ch. Petit-Jean-

Genez (eds.), World Scientific, p. 133

[5] H.F. Beyer, IEEE Transactions on Instr. Meas. 44 (1995) 510

[6]D. McCammon et al., Nucl. Instr. Meth. A326 (1993) 157

[7] R.L. Kelly et al., J. Low Temp. Phys. 93 (1993) 225

[8]D. McCammon, private communication

[9]F. Gatti et al., contribution to this workshop

[10]D. McCammon et al.; and C.K. Stahle et al., contributions to this workshop

[11]C.K. Stahle et al., Nucl. Instr. Meth. A319 (1992) 393

[12]M. Le Gros et al., contribution to this workshop 
Tab. 1: Comparison of experimental and theoretical results on the 1s-Lamb shift (eV) in hydrogen-like $Z=79$ and $Z=92$ systems (taken from [5], see also references in [5]).

\begin{tabular}{lcc}
\hline & $\mathbf{Z}=\mathbf{7 9}$ & $\mathbf{Z}=\mathbf{9 2}$ \\
\hline Experiment: & & \\
$\quad$ Beyer et al. (1995) & $202.3 \pm 7.9$ & $470 \pm 16$ \\
Stöhlker et al. (1993) & & $429 \pm 63$ \\
Briand et al. (1990) & & $520 \pm 130$ \\
Theory: & & \\
Johnson and Soff (1985) & $205.3 \pm 0.5$ & $458.4 \pm 2.7$ \\
Soff (1994) & $203.9 \pm 0.5$ & $463.4 \pm 0.6$ \\
Mohr (1994) & & $464.6 \pm 0.6$ \\
\hline
\end{tabular}

Tab. 2: Comparison of the performance of various detector systems for Lamb shift measurements on hydrogen-like very heavy ions.

\begin{tabular}{|c|c|c|c|}
\hline & \multicolumn{3}{|c|}{ Detector Type } \\
\cline { 2 - 4 } & Ge-Detector & Crystal spectrometer & Calorimeter \\
\hline Energy Resolution & $800 \mathrm{eV}$ & $<50 \mathrm{eV}$ & $30-50 \mathrm{eV}$ \\
Position Resolution & - & - & $0.25-0.5 \mathrm{~mm}$ \\
$\begin{array}{c}\text { Photo Efficiency } \\
(\text { Ex }=\mathbf{5 0} \text { keV })\end{array}$ & $>90 \%$ & - & $\geq 30 \%$ \\
Active Area & $500 \mathrm{~mm}^{2}$ & - & $100-200 \mathrm{~mm}^{2}$ \\
Total Efficiency & $10^{-3}-10^{-4}$ & $10^{-7}$ & $10^{-4}-10^{-5}$ \\
\hline
\end{tabular}




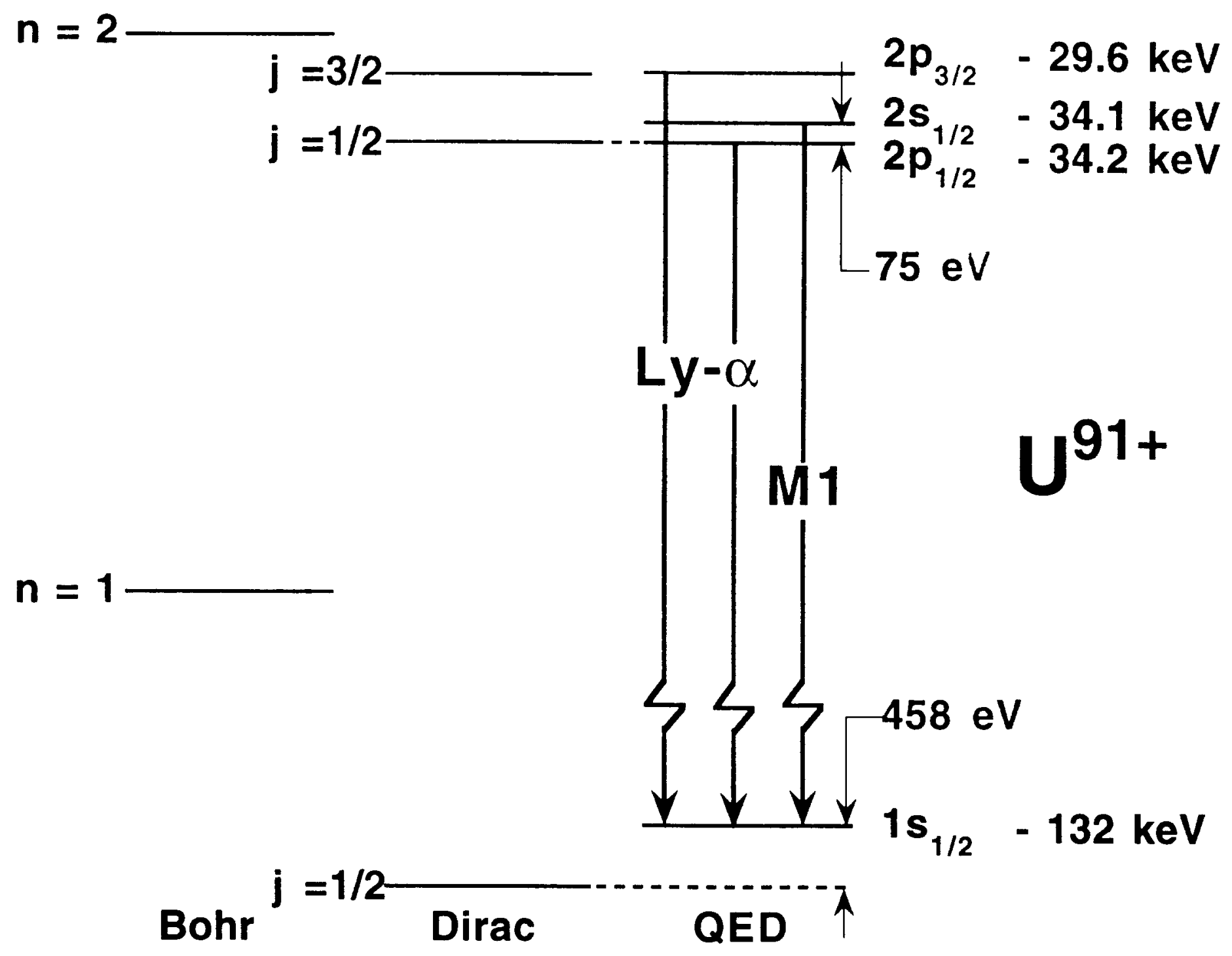

Fig.1: Level scheme for hydrogen-like $\mathrm{U}^{91+}$ according to various atomic models. 


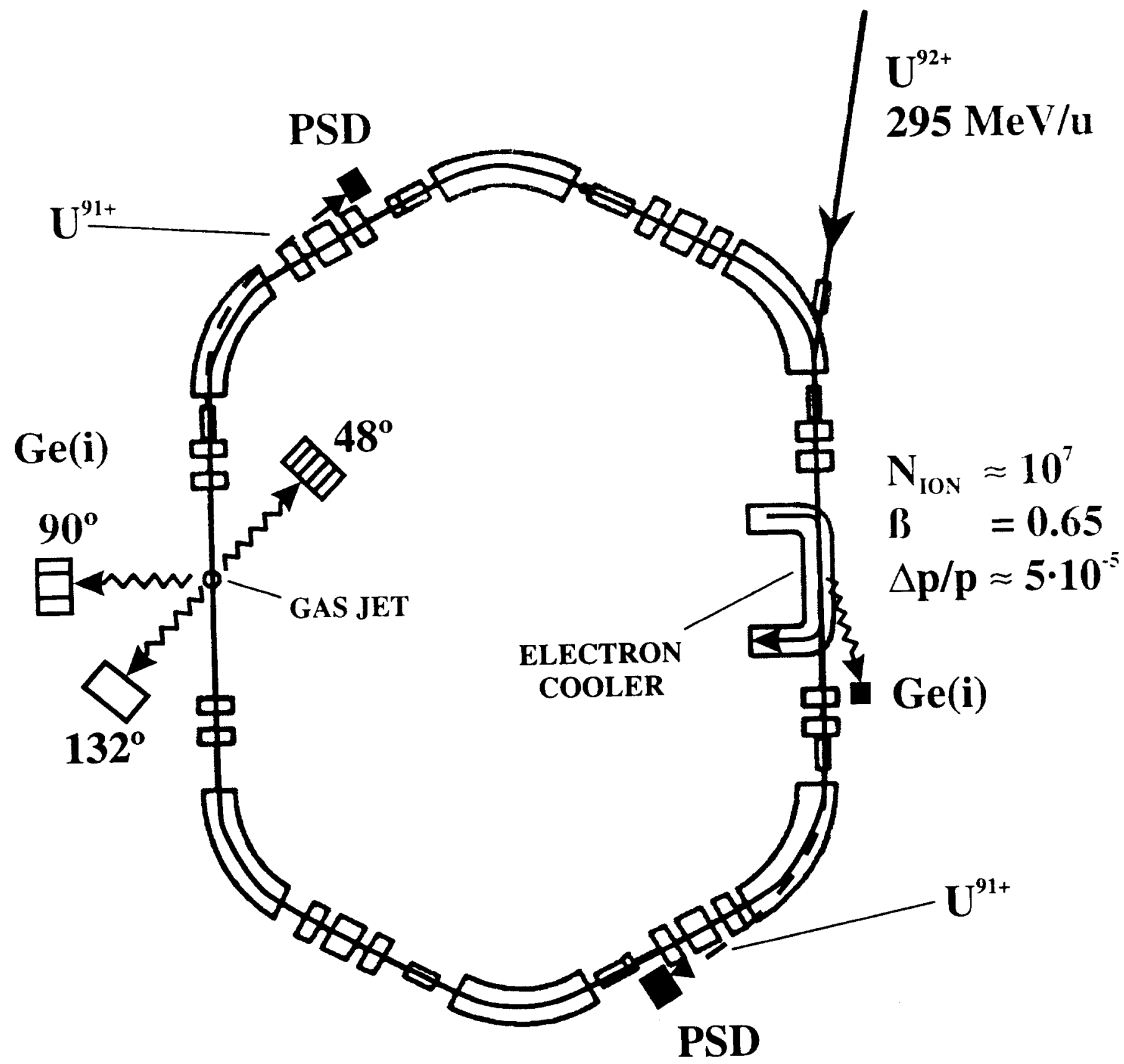

Fig.2: Typical experimental setup for Lamb shift experiments at the internal gas target and at the electron cooler of the storage ring ESR. 